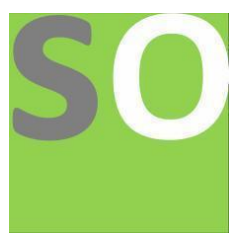

Article title: Soft Set Theoretic on Marriage Problem Predicate Task.

Authors: Frank Appiah[1]

Affiliations: $N / A[1]$

Orcid ids: 0000-0001-6732-9503[1]

Contact e-mail: appiahnsiahfrank@gmail.com

License information: This work has been published open access under Creative Commons Attribution License http://creativecommons.org/licenses/by/4.0/, which permits unrestricted use, distribution, and reproduction in any medium, provided the original work is properly cited. Conditions, terms of use and publishing policy can be found at https://www.scienceopen.com/.

Preprint statement: This article is a preprint and has not been peer-reviewed, under consideration and submitted to ScienceOpen Preprints for open peer review.

DOI: 10.14293/S2199-1006.1.SOR-.PP761HH.v1

Preprint first posted online: 07 April 2021

Keywords: Sentence, Predicate, Soft set, Approximation, Parameters, Value set, Functional value 


\title{
Soft Set Theoretic on Marriage Problem Predicate Task.
}

\author{
Prof Frank Appiah AKA FAEng PhD \\ appiahnsiahfrank@gmail.com \\ 5.04 .2021
}

\begin{abstract}
This report is about theoretical study of soft sets on Marriage Problem Predicate with its tabular representation defined over universe sets, $\mathbf{U}$ with set of parameters from the sentence predicates of the case examples used in the research exploration.
\end{abstract}

Keywords. sentence, predicate name, tabular representation, value set, functional value, soft set, approximation.

\section{Introduction}

Molodtsov [11] defined the soft set in the following way. Let $U$ be an initial universe set and $E$ be a set of parameters. Let $P(U)$ denotes the power set of $U$ and $A$ subset $E$.

DEFINITION 2.1. (See 171.) A pair $(\mathrm{F}, \mathrm{A})$ IS called a soft set over $\mathrm{U}$, where $\mathrm{F}$ is a mapping given by $\mathrm{F}: \mathrm{A}$ implies $\mathrm{P}(\mathrm{U})$.

In other words, a soft set over $U$ is a parametrized family of subsets of the universe $U$. For e member of $A$. $F(E)$ may be considered as the set of e-approximate elements of the soft set $(F, A)$. Clearly, a soft set is not a set. For illustration, Molodtsov considered several examples in [11] one of which is presented below.

EXAMPLE 2.1. Suppose the following.

$U$ is the set of houses under consideration.

$E$ is the set of parameters. Each parameter is a word or a sentence.

$E=$ \{expensive; beautiful; wooden; cheap; in the green surroundings; modern; in good repair; in bad repair\}. 
In this case, to define a soft set means to point out expensive houses, beautiful houses, and so on.

The soft set (F,E) describes the "attractiveness of the houses" which Mr. X (say) is going to buy.

We consider below the same example in more detail for our next discussion.

Suppose that there are six houses in the universe $U$ given by

$\mathrm{U}=\{\mathrm{hl}, \mathrm{h} 2, \mathrm{~h} 3, \mathrm{~h} 4, \mathrm{~h} 5, \mathrm{~h} 6)$ and $\mathrm{E}=\{\mathrm{el}, \mathrm{e} 2, \mathrm{e} 3, \mathrm{e} 4, \mathrm{e} 5)$ :

where

el stands for the parameter 'expensive',

e2 stands for the parameter 'beautiful',

e3 stands for the parameter 'wooden',

e4 stands for the parameter 'cheap',

e5 stands for the parameter 'in the green surroundings'.

Suppose that

$\mathrm{F}(\mathrm{e} 1)=\{\mathrm{h} 1, \mathrm{~h} 4\}$

$F(e 2)=\{h 1, h 3\}$

$F(e 3)=\{h 3, h 4, h 5\}$

$F(e 4)=\{h 1, h 3, h 5\}$

$F(e 5)=\{h 1\}$

The soft set $(F, E)$ is a parametrized family $\left\{F\left(e_{-} i\right), i=1,2,3, . ., 8\right\}$ of subsets of the set $U$ and gives us a collection of approximate descriptions of an object.

I will illustrate the use of predicate sentence from the celebrated Marriage Problem case example to create first tabular representation on soft set and then generate the parametrized family.

Marriage Problem is about sentences or phrases and counting problems. It is logical structured and involves discrete operations like subtraction, addition and multiplication. It is about alphanumeric labeling of sentences or phrases and proofing of combinatorial enumerations. The theory of combinatorics of sentences or phrases or words is called Letter Combinatorics (LC) with 8 bulletin requirements. A Marriage Problem (MP) made up of 5 sentences is used in the exploit of letter combinatorics. A generating function is calculated for MP to handle constraints of arrangement /selection and the combinatorial enumerations of MP. The predicate sentences are made from [5]. This work looks at soft set concepts on and tabular representation of predicates.

The Marriage Problem states that;

(1) Damn it.

(2) What's wrong?

(3) It is a combination of 46 letters.

(4) Akua will not marry you.

(5) Pokua will not marry you.

This research is organised as follows :

- Show the parametrized family of soft set from the initial universe set, 
- A Look again on the predicate sentences with soft set on tabular representation.

\section{Soft Set on Tabular Representation}

The next predicate is to determine if a sentence is a question or not. There is only one question in all the five sentences. It is represented as mpsentenceask predicate sentence. This category predicate is important in this work.

This will take on two passing values of sentence number and an indicator of a question or not. Yes( $\mathrm{Y}$ will be 1$)$ indicates a pass value whiles $\mathrm{No}(\mathrm{N}$ will be 0$)$ does not. The following question stances are:

1. mpsentenceask (1, no).

2. mpsentenceask (2, yes).

3. mpsentenceask (3, no).

4. mpsentenceask (4, no).

5. mpsentenceask (5, no).

General Predicate : mpsentenceask (sentence_no, response).

In generating a set for mpsentenceask (named as MbA), It will give:

$\operatorname{MbA}=\{0,1,0,0,0\}$.

The number of words of a sentence is now represented with mpwordsize predicate sentences. . The following details are as follows :

1. mpwordsize $(1,2)$.

2. mpwordsize $(2,2)$.

3. mpwordsize $(3,6)$.

4. mpwordsize $(4,5)$.

5. mpwordsize $(5,5)$.

This category predicate is important in this work. The set theoretic form is represented as :

$\operatorname{MbWs}=\{1,1,1,1,1\}$.

This predicate took its arguments to be the sentence number and the number of words. General predicate is represented as:

General Predicate : mpwordsize (sentence_no, word_number).

Further details on negation sentences are looked at. This will have the predicate sentence, 
mpnegation. This is explicitly sentences with a not word.

The problem solution are as follows :

1. mpnegation (1, no).

2. mpnegation (2, no).

3. mpnegation $(3$, no $)$.

4. mpnegation (4, yes).

5. mpnegation (5, yes).

General Predicate : mpnegation (sentence_no, response).

The set representation of Mpnegation is

$\mathrm{MbNg}=\{0,0,0,1,1\}$.

MP example has only two negation statements in total. Statements like "damn it" creates a feeling of regret or disappointment. What's wrong did create sudden worry but does not bring the negation that is not interesting. The predicate sentence is represented as mpregret.

These are as follows :

1. mpregret (1, yes).

2. mpregret $(2$, no) .

3. mpregret $(3$, no) .

4. mpregret $(4$, no) .

5. mpregret (5, no).

General Predicate : mpregret (sentence_no, response).

The set theoretical form is given by:

$\operatorname{MbR}=\{1,0,0,0,0\}$

mpworry is the predicate sentence for sudden worry. These includes the following :

- mpworry $(1$, no).

- mpworry $(2$, yes $)$.

- mpworry $(3$, no) .

- mpworry $(4$, no).

- mpworry (5, no).

General Predicate : mpworry (sentence_no, response).

The set theoretical form is given by:

$\mathrm{MbW}=\{0,1,0,0,0\}$.

The problem solver took on statement 3 to bring out an approach. The predicate for this will be mpsolver. The knowledge needed to be programmed are as follows: 
1. mpsolver(1, no).

2. mpsolver(2, no).

3. mpsolver(3, yes).

4. mpsolver (4, no).

5. mpsolver(5, no).

General Predicate : mpsolver (sentence_no, response).

The set theoretical form is given by:

$\operatorname{MbS}=\{0,0,1,0,0\}$

The third round tried to bring out a solution in the context of problem solving. The 4 and 5 statements are involved with names of female sex. These are Akua and Pokua. The fact base for this representation is captured with predicate sentences, mpnamsex. These will include the following :

- mpnamsex $(1$, no).

- mpnamsex $(2$, no).

- mpnamsex $(3$, no).

- mpnamsex $(4, y e s)$.

- mpnamsex (5, yes).

General Predicate : mpnamsex (sentence_no, response).

The set theoretical form is given by:

$\operatorname{MbX}=\{0,0,0,1,1\}$.

Suppose that there are five predicates in the universe $U$ given by $\mathrm{U}=\{\mathrm{N} 1, \mathrm{~N} 2, \mathrm{~N} 3, \mathrm{~N} 4, \mathrm{~N} 5\}$ and $\mathrm{E}=\{\mathrm{MbA}, \mathrm{MbX}, \mathrm{MbS}, \mathrm{MbR}, \mathrm{MbW}, \mathrm{MbNg}\}$ : where

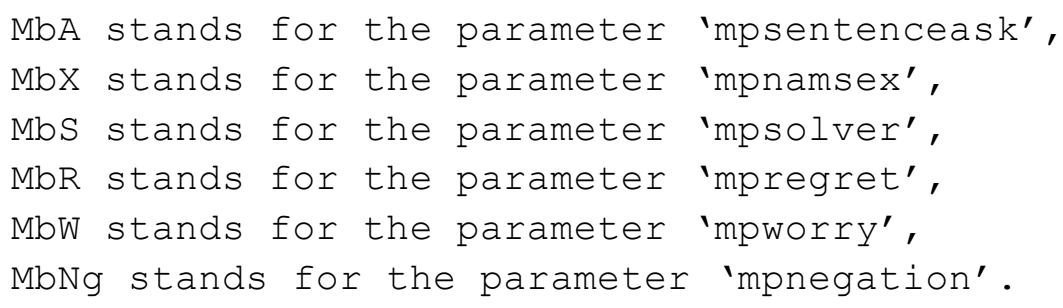

In mapping the power set of the Universe onto the subset of Parameter :

It is suppose that

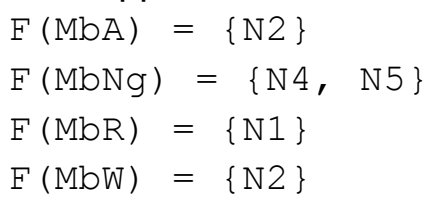


$\mathrm{F}(\mathrm{MbX})=\{\mathrm{N} 4, \mathrm{~N} 5\}$

$\mathrm{F}(\mathrm{MbS})=\{\mathrm{N} 3\}$.

Therefore $\mathrm{F}(\mathrm{MbA})$ means mpsentenceask whose functional value is set $\{\mathrm{N} 2\}$.

Therefore $\mathrm{F}(\mathrm{MbNg})$ means mpnegation whose functional value is set $\{\mathrm{N} 4, \mathrm{~N} 5\}$.

Therefore $F(M b R)$ means mpregret whose functional value is set $\{N 1\}$.

Therefore $F(M b W)$ means mpworry whose functional value is set $\{N 2\}$.

Therefore $F(M b X)$ means mpnamsex whose functional value is set $\{N 4, N 5\}$.

Therefore $F(\mathrm{MbS})$ means mpsolver whose functional value is set $\{\mathrm{N} 3\}$.

We can view the soft set $(F, E)$ as a collection of approximations as shown below: $\{$ mpsentenceask $=\{N 1\}$, mpnamsex $=\{N 4, N 5\}$, mpsolver $=\{N 3\}$, mpregret $=\{N 1\}$, mpworry $=\{\mathrm{N} 2\}$, mpnegation $=\{\mathrm{N} 4, \mathrm{~N} 5\}\}$ where each approximation has two parts:

(i) a predicate $\mathrm{p}$; and

(ii) an approximate value-set v(or simply to be called value-set v)

For example the approximation, "mpsentenceask $=\{\mathrm{N} 1\}$ ", we have the following :

- the predicate name is mpsentenceask and

- the approximate value set or value set is $\{\mathrm{N} 1\}$.

For example the approximation, "mpnamsex $=\{N 4, N 5\}$ ", we have the following :

- the predicate name is mpnamsex and

- the approximate value set or value set is $\{\mathrm{N} 4, \mathrm{~N} 5\}$

For example the approximation, "mpsolver $=\{\mathrm{N} 3\}$ ", we have the following :

- the predicate name is mpsolver and

- the approximate value set or value set is $\{\mathrm{N} 3\}$

For example the approximation, "mpregret $=\{\mathrm{N} 1\}$ ", we have the following :

- the predicate name is mpregret and

- the approximate value set or value set is $\{\mathrm{N} 1\}$

For example the approximation, "mpworry $=\{\mathrm{N} 2\}$ ", we have the following :

- the predicate name is mpworry and

- the approximate value set or value set is $\{\mathrm{N} 2\}$

For example the approximation, "mpnegation $=\{\mathrm{N} 4, \mathrm{~N} 5\}$ ", we have the following :

- the predicate name is mpnegation and

- the approximate value set or value set is $\{\mathrm{N} 4, \mathrm{~N} 5\}$

The following are used in forming soft set on tabular representation :

$\mathrm{MbA}=\{0,1,0,0,0\}$.

$M b X=\{0,0,0,1,1\}$.

$\mathrm{MbS}=\{0,0,1,0,0\}$. 
$M b R=\{1,0,0,0,0\}$

$\mathrm{MbW}=\{0,1,0,0,0\}$.

$\mathrm{MbNg}=\{0,0,0,1,1\}$.

Tabular Representations On Soft Set

\begin{tabular}{|l|l|l|l|l|l|l|}
\hline U & MbA & MbNg & MbR & MbW & MbX & MbS \\
\hline N1 & 0 & 0 & 1 & 0 & 0 & 0 \\
\hline N2 & 1 & 0 & 0 & 1 & 0 & 0 \\
\hline N3 & 0 & 0 & 0 & 0 & 0 & 1 \\
\hline N4 & 0 & 1 & 0 & 0 & 1 & 0 \\
\hline N5 & 0 & 1 & 0 & 0 & 1 & 0 \\
\hline
\end{tabular}

$F(M b A):$ The functional value is in $\operatorname{set}(N 2)=\{1,0,0,1,0,0\}$

$F(M b R):$ The functional value is in $\operatorname{set}(\mathrm{N} 1)=\{0,0,1,0,0,0\}$

$F(M b W):$ The functional value is in $\operatorname{set}(N 2)=\{1,0,0,1,0,0\}$

$F(\mathrm{MbNg})$ : The functional value is in $\operatorname{set}(\mathrm{N} 4, \mathrm{~N} 5)=\{0,1,0,0,1,0\}$

$F(M b X)$ : The functional value is in $\operatorname{set}(N 4, N 5)=\{0,1,0,0,1,0\}$

$F(M b S)$ : The functional value is in $\operatorname{set}(N 3)=\{0,0,0,0,0,1\}$

\section{Conclusion}

This work on tabular representation and soft computing concludes with the following remarks:

- Six soft response set are achieved.

- Table representation of the soft set is achieved.

- Look at approximation in terms of predicate name and approximate value set is achieved.

- The universe of marriage Problem Predicate is achieved. 


\section{Further Reading.}

(1) Appiah Frank. Letter Combinatorics : Theory on counting problems. EPSRC UK Turing Al Letter. 2020

(2) Appiah Frank. Letter Combinatorics : Theory on counting problems. Marriage Problem. Mendeley Publication. 2020

(3) Hooper, Joan B. On assertive predicates.In Syntax and Semantics volume 4, pp.

91-124. Brill, 1975.

(4) Yoon, Youngeun. Total and partial predicates and the weak and strong interpretations. Natural language semantics 4, no. 3 (1996): 217-236.

(5) Frank Appiah. Representative Artificials On LetterCombinatorics Case With Predicate Sentences. Easychair No 4556. November 13, 2020.

(6) Jech, T. (2013). Set theory. Springer Science \& Business Media.

(7) Fraenkel, A. A., Bar-Hillel, Y., \& Levy, A. (1973). Foundations of set theory. Elsevier.

(8) Enderton, H. B. (1977). Elements of set theory. Academic press.

(9) Number Unit Conversion. 2021. Accessed Online:

http://www.unitconversion.org/numbers/binary-to-base-10-conversion.html

(10) String Conversion. 2021. Accessed Online :

https://www.convertbinary.com/text-to-binary/

(11) D. Molodtsov, Soft set theory-First results, Computers Math. Applic. 37 (4/5), 19-31, (1999) 